REVIEW ARTICLE

J.I. Lane

R.J. Witte

B. Bolster

M.A. Bernstein

K. Johnson

J. Morris

\title{
State of the Art: 3T Imaging of the Membranous Labyrinth
}

SUMMARY: This article reviews the anatomy of the membranous labyrinth and demonstrates the ability of high-resolution MR imaging at $3 \mathrm{~T}$ to visualize the neurosensory epithelium by using the latest fast spin-echo techniques.

V isualization of the membranous structures of the inner ear has been limited to the detection of normal fluid signal intensity within the bony labyrinth on $1.5 \mathrm{~T}$ or $3 \mathrm{~T} \mathrm{MR}$ imaging. The ability to directly visualize the endolymphatic vesicles of the inner ear might permit the development of imaging criteria in the diagnosis and treatment of labyrinthine disease. Currently 3D sequences to include constructive interference in the steady state (CISS) or fast spin-echo (FSE) techniques provide excellent depiction of the nerves within the internal auditory canal as well as the fluid within the labyrinth, but attempts to image organs of the membranous labyrinth have been compromised by susceptibility banding artifact in the case of gradient-echo acquisitions and image blurring inherent to fast spin-echo (FSE) sequences. ${ }^{1}$ In this article, we review the anatomy of the inner ear and describe the utility of 3D variable flip-angle FSE (3D VFA FSE) technique in demonstrating some of the more prominent neurosensory components of the membranous labyrinth at $3 \mathrm{~T}$.

\section{Imaging Technique}

When imaging the labyrinth, it is best to take advantage of the increased signal intensity to noise and improved resolution offered by $3 \mathrm{~T}$. The images presented in this article were all acquired on a 3T MR imaging system (Magnetom Trio [TIM System]; Siemens Medical Solutions, Erlangen, Germany) by using $7-\mathrm{cm}$ loop coils placed over the external auditory canals of several healthy volunteers. The 3D VFA FSE technique was used in the standard axial and coronal planes in addition to 2 oblique planes acquired along the oblique sagittal (Poschl) and oblique coronal (Stenvers) plane. Images were acquired at a 6-cm FOV, $202 \times 256$ matrix, and $0.32-\mathrm{mm}$ partitions, yielding nearly $0.3-\mathrm{mm}$ isotropic spatial resolution. Images were further interpolated in-plane to approximately $0.14 \times 0.12 \mathrm{~mm}$. TR and effective TE were 1400 $\mathrm{ms}$ and $131 \mathrm{~ms}$, respectively, yielding an acquisition time of approximately 11.5 minutes. Reference was made to an ex vivo T2weighted dataset of a cadaver temporal bone specimen scanned at 9.4T Oxford vertical bore (Oxford Instruments, Oxford, UK) for positive correlative identification of membranous labyrinthine structures. The technique used to acquire this ex vivo dataset has been previously published. ${ }^{2}$

In VFA FSE pulse sequences, the flip angle of the refocusing pulses is not fixed at a constant value (eg, $180^{\circ}$ ) but is instead

From the Department of Radiology (J.I.L., R.J.W., M.A.B., J.M.), Mayo Clinic, Rochester, Minn; and Siemens Medical Solutions (B.B., K.J.), Malvern, Pa.

Please address correspondence to John Lane, MD, Department of Radiology, Mayo Clinic, 200 First St SW, Rochester, MN 55902; e-mail: lane.john@mayo.edu

DOI 10.3174/ajnr.A1036

modulated according to a predetermined schedule, which is calculated on the basis of the desired image contrast properties. ${ }^{3-7}$ The use of the VFA evolution has several important benefits for temporal bone imaging. As designed, this technique has the effect of reducing the change in signal intensity through the echo train, allowing longer echo trains and thus enabling a high-resolution single slab isotropic acquisition in a clinically feasible scanning time. This more consistent echo-to-echo signal intensity has the additional benefit of reducing blurring, especially in tissues with relatively short T2. Further, because smaller flip angles are used, the radiofrequency heating as measured by the specific absorption rate (SAR) is substantially reduced, enabling applications at 3T without the acquisition time penalty normally associated with standard FSE technique at that field strength.

For the acquisitions in this article, a pseudo-steady-state flip angle evolution at $130^{\circ}$ was chosen to obtain high signal to noise required for higher spatial resolution. By using this schedule, the flip angle quickly transitions to and maintains a flip angle of $130^{\circ}$ for each echo train. This allowed relatively long echo trains (37 for these acquisitions) while staying within clinically allowed SAR limits.

\section{Clinical Anatomy}

The inner ear contains the neurosensory organs of hearing and equilibrium, receiving the terminal distributions of the audi-

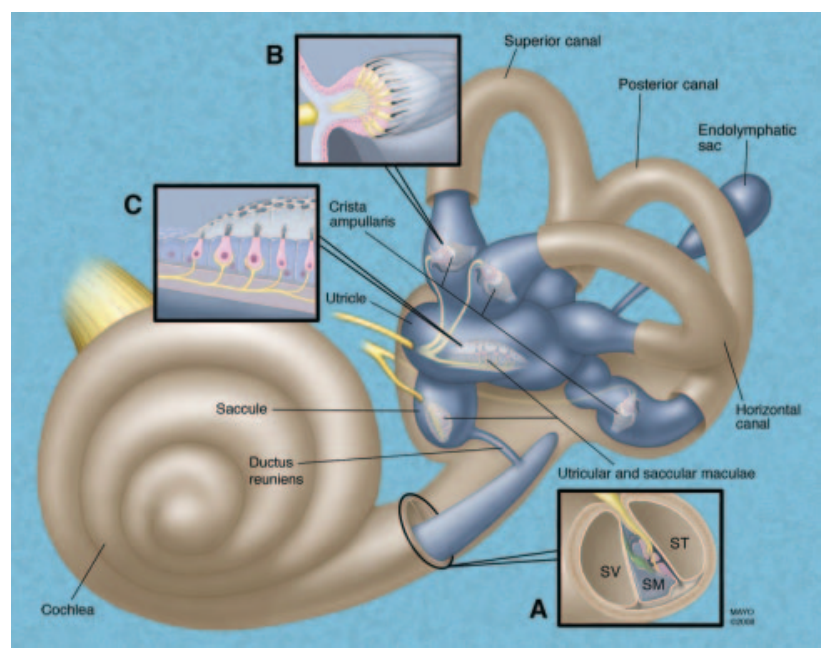

Fig 1. Schematic representation of the membranous labyrinth. $A$, magnified cross-section of the cochlea. $B$, Magnified exposed view of the crista ampullaris of the superior semicircular duct. $C$, Magnified exposed view of the utricular macula. SV indicates scala vestibuli; SM, scala media; ST, scala tympani. 

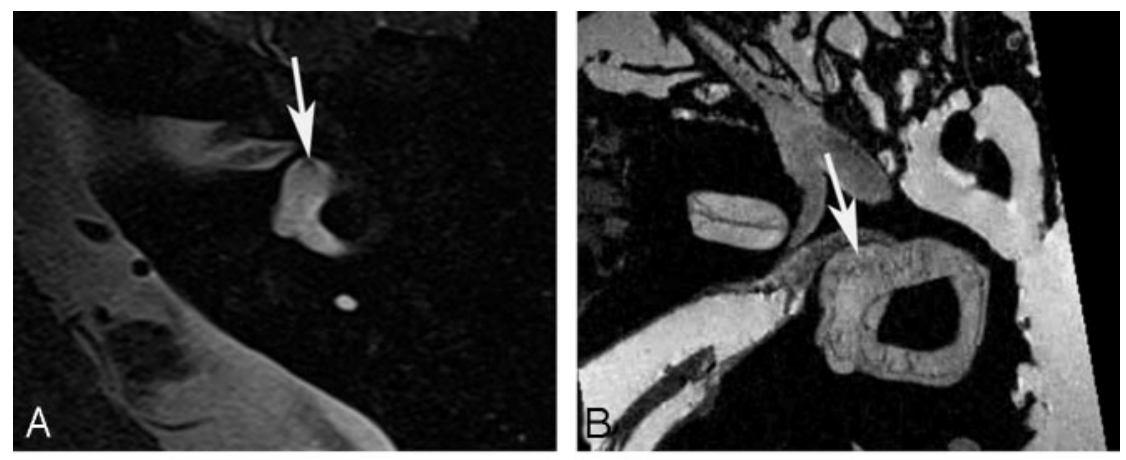

Fig 2. Utricular macula. Axial $(A$ and $B)$, coronal $(C$ and $D)$, oblique sagittal (Poschl plane) ( $E$ and $F$ ), and oblique coronal (Stenvers plane) $(G$ and $H$ ) images of the utricular macula. In vivo 3T $(A, C, E$, and $G)$ and ex vivo 9.4T $(B, D, F$, and $H) \mathrm{MR}$ images demonstrate low-signal-intensity focus (arrow) corresponding to the neurosensory epithelium of the utricular macula.
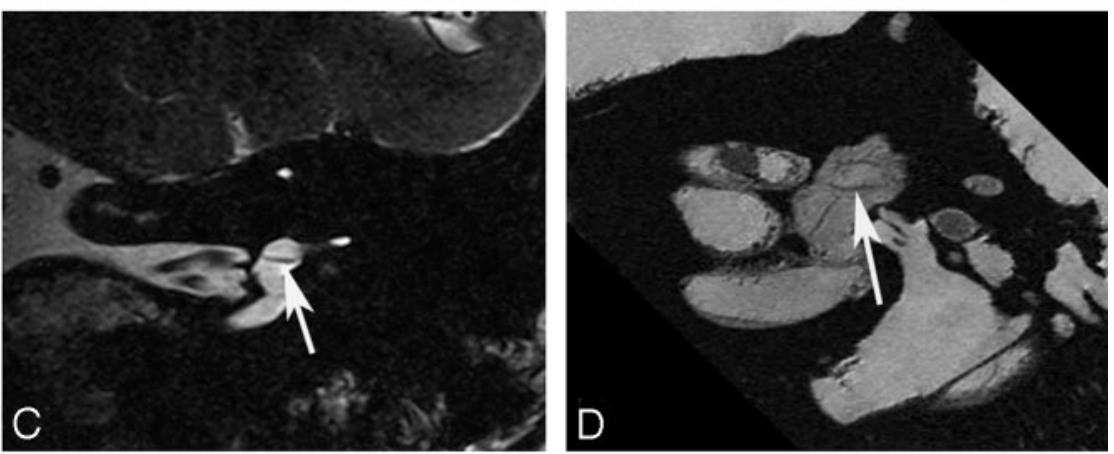

epithelium of these endolymphatic vesicles.

The membranous structures of the bony vestibule do not quite preserve the form of the osseous cavity that contains them but consist of 2 membranous sacs, the utricle and the saccule. These structures contain the sensory organs that detect linear acceleration. The anterior floor of the utricle is focally thickened, consisting of thousands of otoliths floating on a gelatinous membrane beneath the neurosensory hair cells, which receive the utricular filaments of the superior vestibular nerve. This thickened area is the utricular macula. The cavity of the utricle communicates with the semicircular ducts by 5
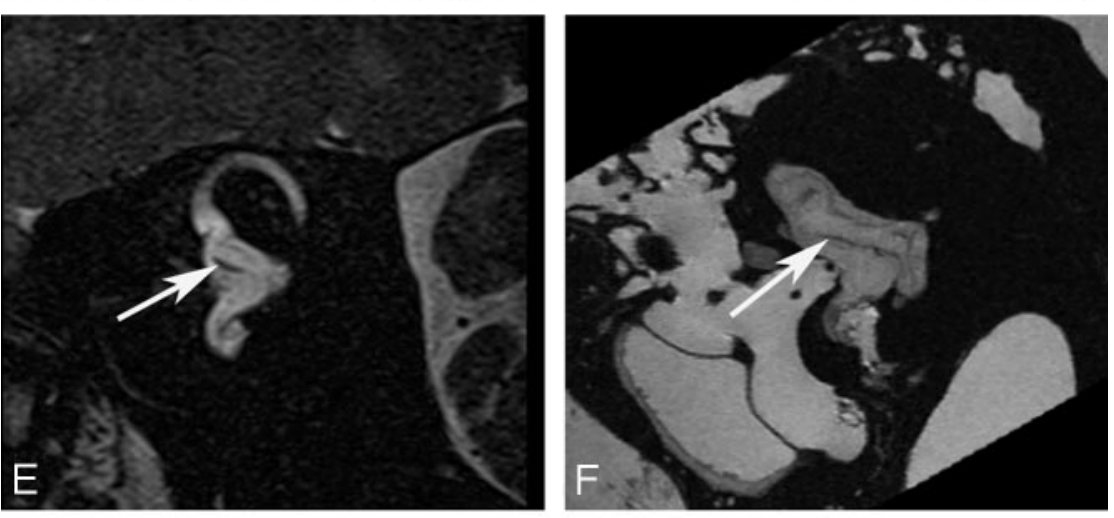
orifices. It also communicates with the endolymphatic duct and saccule by way of the utriculosaccular duct.

The saccule is the smaller of the 2 vestibular sacs and lies in the spherical recess of the medial wall of the bony vestibule near the opening of the scala vestibuli of the cochlea. The medial wall of the saccule exhibits an ellipsoid thickening, the saccular macula (identical in composition to the utricular macula), innervated by the saccular branch of the inferior vestibular nerve and oriented approximately $90^{\circ}$ to the plane
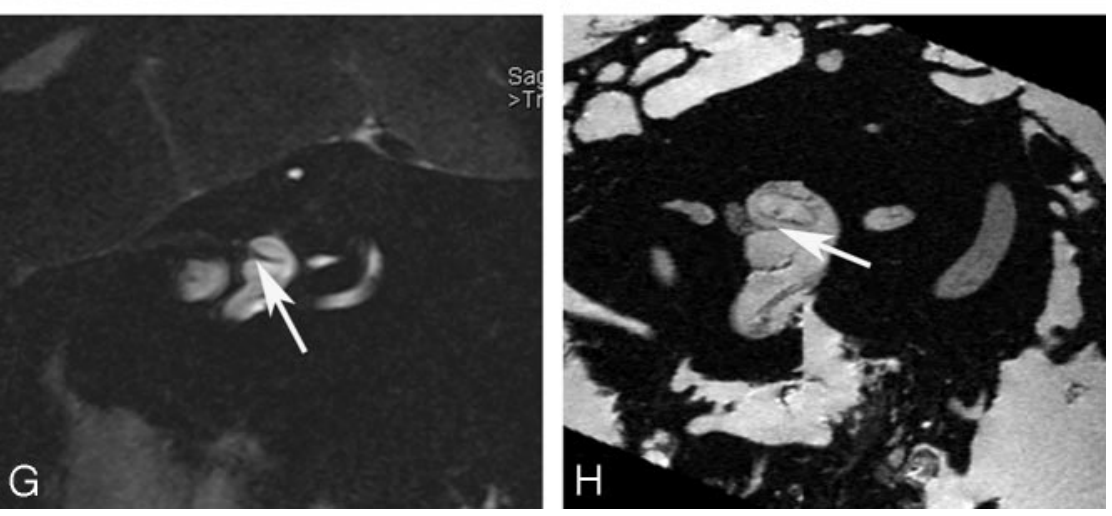
of the utricular macula. The endolymphatic duct, arising from the posterior wall of the saccule, joins the utriculosaccular duct and passes through the vestibular aqueduct to end in the endolymphatic sac on the posterior surface of the petrous bone, beneath the dura mater. The ductus reuniens arises from the floor of the saccule and opens into the cochlear duct near its vestibular end.

The semicircular ducts occupy approximately one fourth of the cross-sectional area of the osseous canals but are of similar form, each with an ampullated end. They open by 5 orifices into the utri-

tory and vestibular nerves. It is known as the labyrinth, because of the complexity of its shape and consists of 2 parts: the osseous labyrinth, the cavity within the petrous bone, and the membranous labyrinth, a series of communicating membranous sacs and ducts contained by the osseous cavity (Fig 1).

The membranous labyrinth is partly separated from the bony walls by the perilymph. In certain places, it is fixed to the walls of the cavity. The sacs and ducts of the membranous labyrinth are filled with endolymph, and branches of the acoustic and vestibular nerves terminate within the sensory cle, 1 opening being common to the posterior end of the superior duct and the superior end of the posterior duct (the common crus). In the ampullae, the wall is thickened, containing a fiddle-shaped transversely oriented complex of hair cells, the crista ampullaris. These sensory organs detect angular (ie, rotational) acceleration. The superior and horizontal ampullae are innervated by the superior vestibular nerve, and the posterior ampulla is innervated by the posterior (singular) branch of the inferior vestibular nerve.

The utricle, saccule, and semicircular ducts are held in po- 

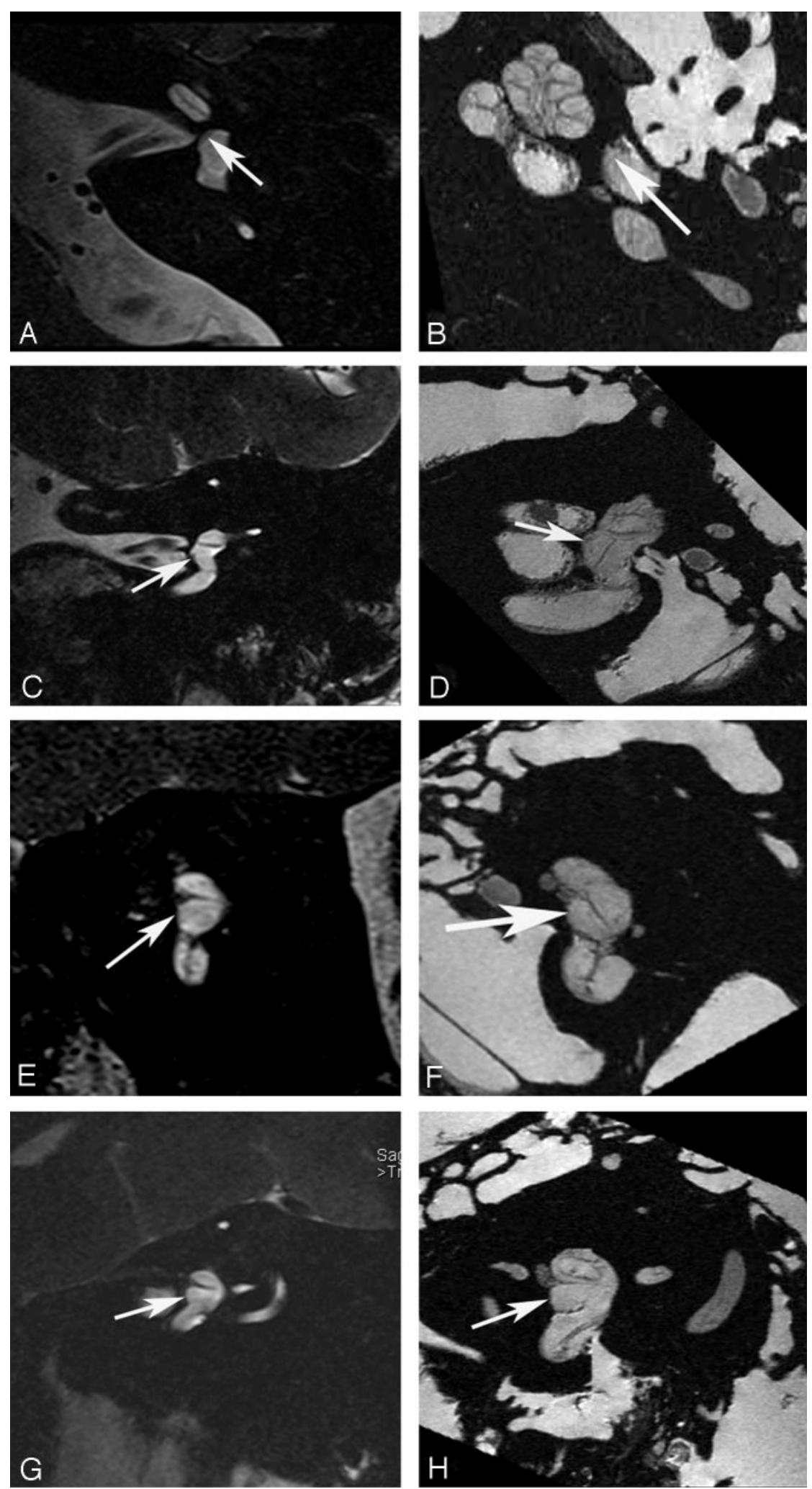

Fig 3. Saccular macula. Axial $(A$ and $B)$, coronal $(C$ and $D)$. oblique sagittal (Poschl plane) ( $E$ and $F$ ), and oblique coronal (Stenvers plane) $(G$ and $H$ ) images. Ex vivo 9.4T MR images $(B, D, F$, and $H)$ demonstrate low-signal-intensity curvilinear focus (arrow) corresponding to the neurosensory epithelium of the saccular macula along the medial wall of the vestibule. In vivo $3 T \mathrm{MR}$ images $(A, C, E$, and $G)$ do not differentiate the macula from the medial wall of the vestibule (arrow).

The cochlear duct (scala media) consists of a spirally arranged tube enclosed in the bony canal of the cochlea and lying along its outer wall. The osseous spiral lamina extends only part of the distance between the modiolus and the outer wall of the cochlea; the basilar membrane extends from its free edge to the outer wall, completing the partition that separates the scala media ventrally from the scala tympani dorsally. A more delicate Reissner membrane extends from the thickened periosteum covering the osseous spiral lamina to the outer wall of the cochlea, where it attaches a short distance above the outer edge of the basilar membrane. The Reissner membrane separates the scala vestibuli ventrally from the scala media dorsally. Contained within the cochlear duct (scala media) is the spiral organ of Corti, the auditory apparatus composed of the sensory hair cells, innervated by branches of the cochlear nerve.

\section{Imaging Anatomy}

The membranes that contain the endolymph of the inner ear are difficult to appreciate even when imaging ex vivo at 9.4T. However, there are distinct sites of thickening where the neurosensory epithelium invests the walls of the membranous structures of the inner ear that may permit their visualization on clinical imaging. This is most apparent when attempting to image the utricular macula, which is best seen in the oblique sagittal (Poschl plane) or direct coronal planes as a linear area of low signal intensity (Fig 2). It is actually more prominent on in vivo images than on ex vivo images at higher field strengths because the otoliths that carpet the macula often become dislodged during the preparation process. ${ }^{3,4}$ The hypointense utricular macula is out-

sition by numerous fibrous bands, identical to the arachnoid membrane, which stretch from the bony walls across the perilymphatic space.

The cochlea consists of 1 endolymphatic lumen (cochlear duct) and 2 perilymphatic lumina: the scala vestibuli, which transmits the acoustic fluid wave from the vestibule, and the scala tympani, which allows the fluid wave to decompress at the round window membrane. lined by hyperintense endolymph that fills the utricular lumen superiorly and the saccule inferiorly. It has a single point of attachment along the anterior wall of the vestibule. ${ }^{5}$ The saccular macula is more difficult to appreciate because its entire surface is applied closely to the inferomedial wall of the vestibule (Fig 3). It too is carpeted with otoliths, but because only its lateral surface is in contact with endolymph (the medial surface being applied closely to the bony wall of the vestibule), 

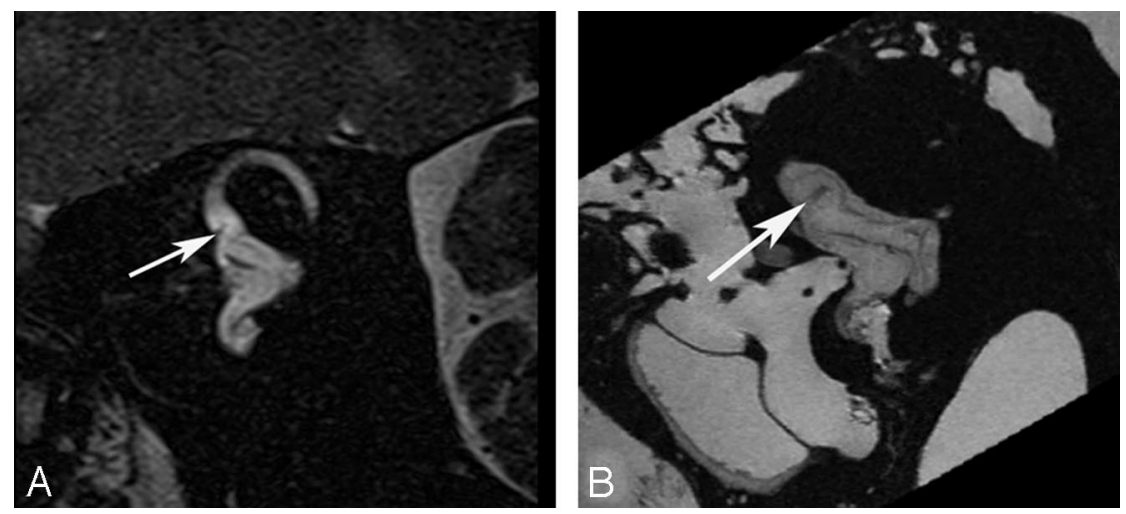

Fig 4. Crista ampullaris of the superior semicircular duct. $A$ In vivo 3T oblique sagittal (Poschl plane) image in the plane of the superior canal demonstrates a linear area of low signal intensity (arrow) corresponding to the crista ampullaris of the superior duct. $B$, Ex vivo 9.4T oblique sagittal image demonstrates similar findings (arrow).
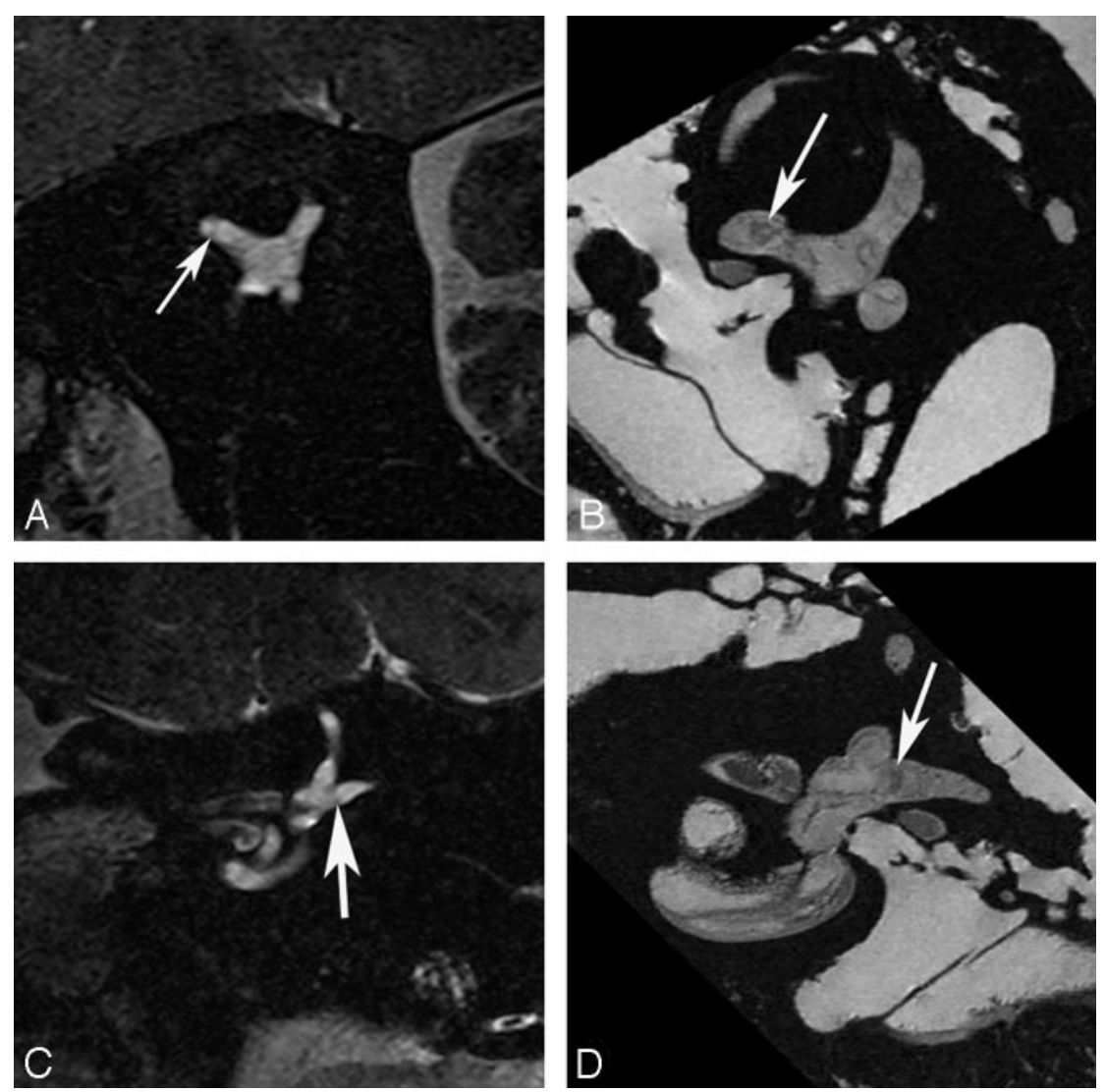

Fig 5. Crista ampullaris of the lateral semicircular duct. $A$, In vivo $3 \mathrm{~T}$ oblique sagittal (Poschl plane) image in the plane of the superior canal demonstrates a linear area of low signal intensity (arrow) corresponding to the crista ampullaris of the lateral duct. $B$, Ex vivo 9.4T oblique sagittal image demonstrates similar findings (arrow). $C$, In vivo 3 T coronal image demonstrates focal area of low signal intensity (arrow) in the ampulla of the lateral canal corresponding to the crista. $D$, Corresponding ex vivo 9.4T image with a similar appearance (arrow).

techniques. ${ }^{2}$ These studies have permitted a better understanding of the configuration of these membranous structures but are prone to dissection and preparation artifacts inherent to ex vivo examinations. The ability to visualize these structures in vivo presents opportunities to advance our understanding of the inner ear in both normal and diseased states.

T2-weighted 3D sequences of the labyrinth and internal auditory canal have become a standard in MR imaging of patients with vestibulocochlear symptoms. These sequences are based on either CISS or FSE technique. Both techniques have inherent shortcomings when it comes to imaging the small structures of the membranous labyrinth. Balanced-SSFP sequences are compromised by susceptibility artifact at air-

it is not outlined by fluid as is the utricular macula. ${ }^{5}$ The ampullated ends of the semicircular ducts contain the crista ampullaris (also known as the transverse septum), the neurosensory organ of the duct. This flame-shaped structure runs perpendicular to the long axis of the duct. It does not contain otoliths and, therefore, is less perceptible on diagnostic MR imaging. We have found the oblique sagittal (Poschl plane) and direct coronal planes to be the best at imaging the superior and horizontal ampullae (Figs 4 and 5) and the oblique sagittal, best for the posterior ampulla (Fig 6).

Although the cochlear duct, containing the neurosensory organ of Corti, can be clearly resolved at $9.4 \mathrm{~T}$ in a cadaver specimen, we have been unsuccessful in our attempts to identify it at $3 \mathrm{~T}$ by using this technique (Fig 7).

\section{Discussion}

Demonstration of the membranous structures of the human inner ear has largely been accomplished ex vivo by using traditional histopathologic techniques or, more recently, with microimaging bone and bone-soft tissue interfaces, which can be particularly problematic at the skull base and accentuated at 3T. Although some of this artifact can be reduced by summing successive phase-cycled acquisitions reconstructed with a maximum intensity projection as with CISS, these artifacts again become problematic with the very small FOVs required for temporal bone imaging and obscure portions of the membranous labyrinth. ${ }^{1}$ FSE sequences have image blurring that can also be amplified at 3T. ${ }^{1}$

The 3D VFA FSE technique can be used clinically to reduce both susceptibility artifact and image blurring. ${ }^{6-9}$ Imaging acquisitions presented in this article were conducted in volunteers; therefore, image resolution has yet to be duplicated in a clinical cohort. Our results indicate that it is possible to visualize portions of the membranous labyrinth that may be affected by vestibulocochlear pathology. Disease processes that are known to produce endolymphatic hydrops (ie, Meniere disease) and clinically 

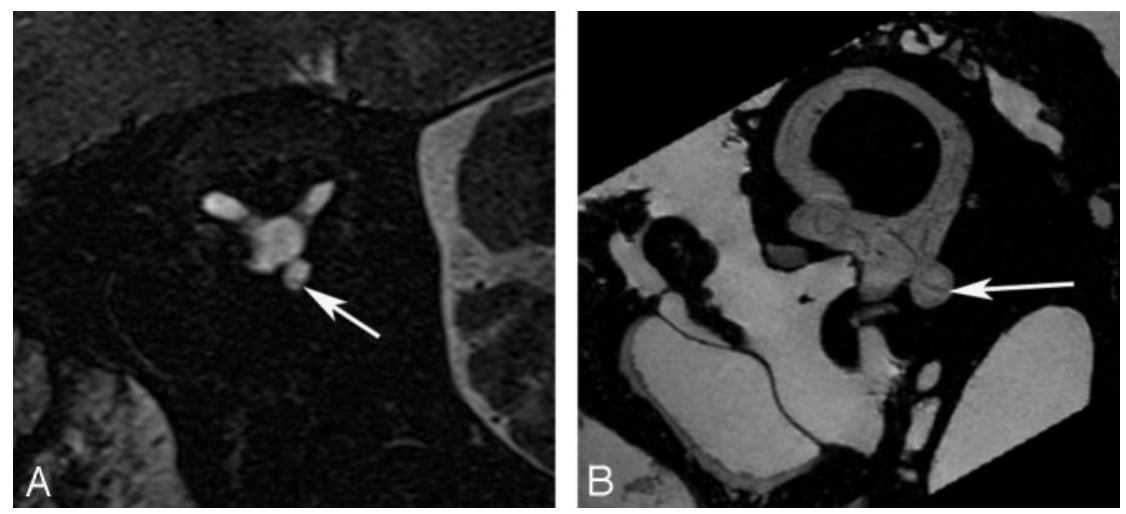

Fig 6. Crista ampullaris of the posterior semicircular duct. $A$ In vivo 3T oblique sagittal (Poschl plane) image in the plane of the superior canal demonstrates a linear area of low signal intensity (arrow) corresponding to the crista ampullaris of the posterior duct. $B$, Ex vivo 9.4T oblique sagittal image demonstrates similar findings (arrow)
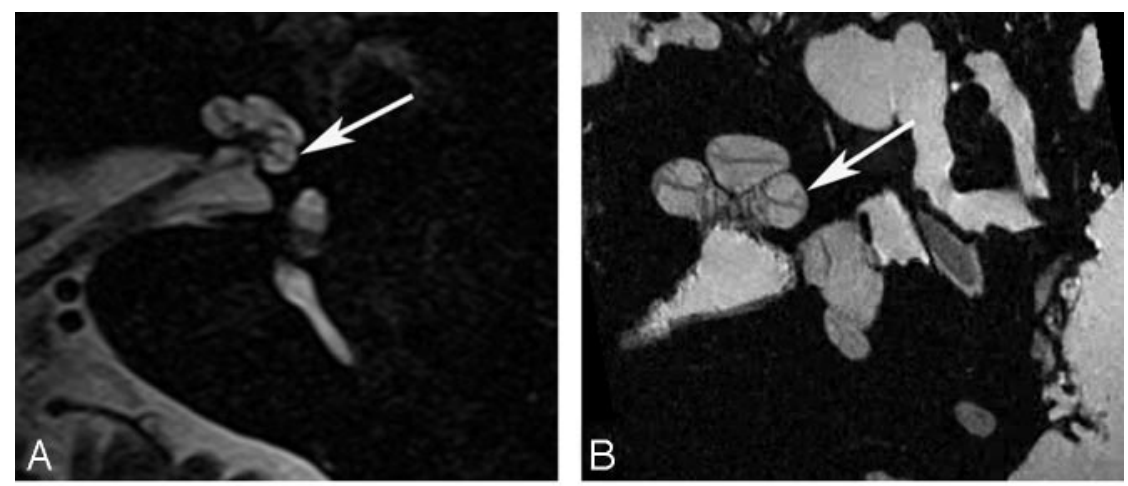

Fig 7. Cochlear duct. $B$ and $D$, Ex vivo 9.4T MR images in the axia and oblique sagittal (Poschl) plane demonstrate the triangular fluid compartment in the peripheral aspect of the basal turn of the cochlear (arrom), corresponding to the cochlear duct. $A$ and $C$, corresponding in vivo 3T MR images do not adequately resolve the Reissner membrane and, therefore, do not demarcate the perilymph in the scala vestibuli from the endolymph in the cochlear duct. Approximated location of the duct is indicated (arrow).

contrast to diffuse from the CSF to the perilymph ${ }^{11}$ or by introduction of dilute gadolinium into the middle ear with diffusion of contrast into the perilymph across the round window membrane. ${ }^{12}$ Both these techniques
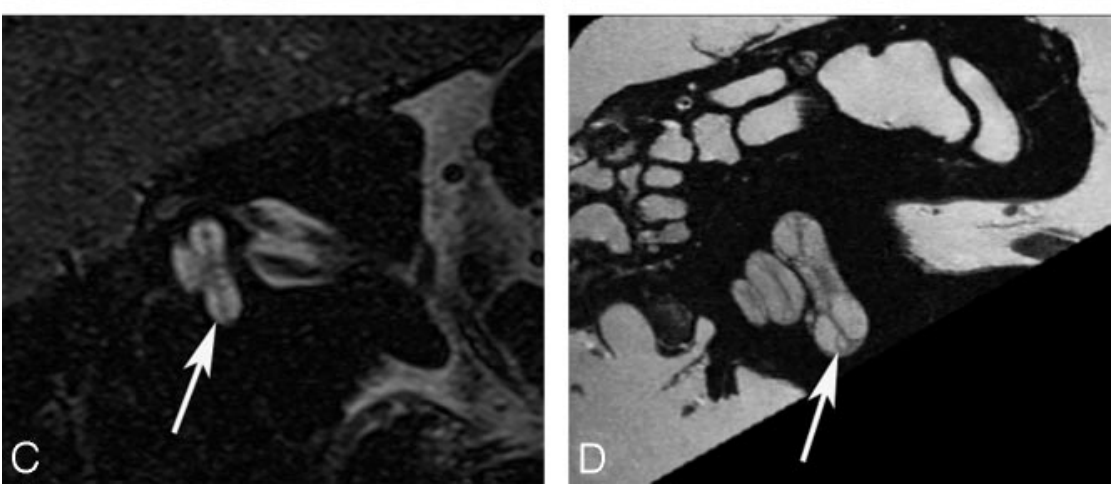
would be difficult to introduce into clinical practice for obvious reasons.

\section{Conclusions}

3T MR imaging of the temporal bone by using the VFA FSE technique can achieve limited resolution of the membranous structures of the inner ear. It is our hope that this pictorial review of inner ear anatomy will prove useful in developing advanced clinical imaging protocols for the assessment of cochleovestibular disease.

present with hearing loss and vestibular symptoms will often have normal findings on MR imaging by using standard imaging protocols. The ability to visualize and consistently localize the position of the utricular macula within the vestibule in healthy volunteers is encouraging. Endolymphatic hydrops will more commonly affect the cochlear duct and saccule, leading to varying degrees of saccular distention. This may cause displacement and elevation of the overlying utricular macula, particularly because the macula has a single point of fixation to the anterior wall of the vestibule. ${ }^{5}$ The ability to detect utricular macular displacement may provide the opportunity to develop imaging criteria for the diagnosis of endolymphatic hydrops, a diagnosis that, to date, remains one of exclusion from an imaging standpoint. Further study regarding the utility of detecting displacement of the utricular macula in cases of hydrops is certainly warranted.

Current limits of resolution do not permit visualization of the cochlear duct (scala media) by using this VFA FSE technique.

Other techniques have been presented and published, which attempt to opacify the perilymph with gadolinium to outline the endolymphatic structures by using a 3D fluid-attenuated inversion recovery technique. This can be done with triple-dose intravenous injection with delayed scanning (optimal at 4-8 hours), allowing

\section{References}

1. Lane JI, Ward H, Witte RJ, et al. 3-T imaging of the cochlear nerve and labyrinth in cochlear-implant candidates: 3D fast recovery fast spin-echo versus $3 \mathrm{D}$ constructive interference in the steady state techniques. AJNR Am J Neuroradiol 2004;25:618-22

2. Lane JI, Witte RJ, Henson OW, et al. Imaging microscopy of the middle and inner ear: Part II: MR microscopy. Clin Anat 2005;18:409-15

3. Schuknecht HF. Pathology of the Ear. Boston: Harvard University Press; 1976

4. Wright CG, Hubbard DG. Observations of otoconial membranes from human infants. Acta Otolaryngol 1978;86:185-94

5. Uzun-Coruhlu H, Curthoys IS, Jones AS. Attachment of the utricular and saccular maculae to the temporal bone. Hearing Res 2007;233:77-85. Epub 2007 Aug 25

6. Le Roux P, Hinks RS. Stabilization of echo amplitudes in FSE sequences. Magn Reson Med 1993;30:183-90

7. Alsop DC. The sensitivity of low flip angle RARE imaging. Magn Reson Med 1997;37:176-84

8. Mugler JPIII, Kiefer B, Brookeman JR. Three-dimensional T2-weighted imaging of the brain using very long spin-echo trains. Presented at: 8th Annual Scientific Meeting of International Society of Magnetic Resonance in Medicine, Denver, Colo, April 1-7, 2000

9. Hennig J, Weigel M, Scheffler K. Multiecho sequences with variable refocusing flip angles: optimization of signal behavior using smooth transitions between pseudo steady states (TRAPS). Magn Res Med 2003;49:527-35

10. Busse RF, Hariharan $\mathrm{H}, \mathrm{Vu} \mathrm{A}$, et al. Fast spin echo sequences with very long echo trains: design of variable refocusing flip angle schedules and generation of clinical T2 contrast. Magn Res Med 2006;55:1030-37

11. Naganawa S, Komada T, Fukatsu H, et al. Observation of contrast enhancement in the cochlear fluid space of healthy subjects using a 3D-FLAIR sequence at 3 Tesla. Eur Radiol 2006;16:733-37

12. Nakashima T, Naganawa S, Sugiura M, et al. Visualization of endolymphatic hydrops in patients with Meniere's disease. Laryngoscope 2007;117:415-20 